\title{
FREE SUBGROUPS OF QUATERNION ALGEBRAS
}

\author{
ROGER C. ALPERIN
}

(Communicated by Ron Solomon)

\begin{abstract}
Using the theory of group actions on trees, we shall prove that if a quaternion algebra over Laurant polynomials is not split then a certain congruence subgroup of the group of norm one elements is a free group. This generalizes and gives an easy, conceptually simpler proof than that given by Pollen for the field of real numbers.
\end{abstract}

Suppose that $F$ is a field; with elements $\alpha$ and $\beta$ we construct the quaternion algebra $\mathscr{H}$ over $F$ with basis $1, I, J$, and $K$ so that $I^{2}=\alpha, J^{2}=\beta$, and $K=I J=-J I$. The involution $\sigma$ on $\mathscr{H}$ is defined by $I \rightarrow-I, J \rightarrow-J$, $K \rightarrow-K$ and extended linearly to all of $\mathscr{H}$ by the identity on $F$. The norm $\mathscr{N}$ is defined by $\mathscr{N}(x)=x \sigma(x)$. It is easy to see that $\mathscr{N}(x y)=\mathscr{N}(x) \mathscr{N}(y)$, so that the elements of norm one in this algebra form a group, $\mathscr{G}(F ; \alpha, \beta)$. By extending the field of scalars to the function field $F(t)$, and extending the involution $\sigma$ by $t \rightarrow t^{-1}$, we obtain the algebra $\mathscr{H}(t)$. We denote the norm one group for this algebra by $\mathscr{G}(F(t) ; \alpha, \beta)$. There is a natural representation of the quaternion algebra to the matrix algebra of degree two over the field $F(\sqrt{\alpha})$ via

$$
I \rightarrow\left[\begin{array}{cc}
\sqrt{\alpha} & 0 \\
0 & -\sqrt{\alpha}
\end{array}\right] \text { and } J \rightarrow\left[\begin{array}{ll}
0 & 1 \\
\beta & 0
\end{array}\right] .
$$

In this representation the element $x=a 1+b I+c J+d K$ is represented by the matrix

$$
\left[\begin{array}{cc}
a+b \sqrt{\alpha} & c+d \sqrt{\alpha} \\
\beta(c-d \sqrt{\alpha}) & a-b \sqrt{\alpha}
\end{array}\right] .
$$

The norm is represented by the determinant, so that $\mathscr{N}(x)=r \sigma(r)-\beta s \sigma(s)$, where $r=a+b \sqrt{\alpha}, s=c+d \sqrt{\alpha}$, with $\sigma(\sqrt{\alpha})=-\sqrt{\alpha}$. The representation is extended similarly to $\mathscr{H}(t)$.

In this article we shall consider the subgroups where the coefficient rational functions are in the ring of Laurant polynomials, $\mathscr{G}\left(F\left[t, t^{-1}\right] ; \alpha, \beta\right)$, and its congruence subgroup $\Gamma$ which is the kernel of the homomorphism

$$
\mathscr{G}\left(F\left[t, t^{-1}\right] ; \alpha, \beta\right) \rightarrow \mathscr{G}(F ; \alpha, \beta)
$$

Received by the editors August 13, 1991.

1991 Mathematics Subject Classification. Primary 20 E08.

Key words and phrases. Free group, Bruhat-Tits tree, quaternion algebra.

Research supported by NSA. 
via $t \rightarrow 1$. In the representation above this means that $r, s$ are Laurant polynomials over $F(\sqrt{\alpha})$, and $r(1)=1, s(1)=0$. Let $L=F(\sqrt{\alpha})$ and $A=L\left[t, t^{-1}\right]$.

Theorem 1. Any subgroup of $\Gamma$ that has no nontrivial unipotents is a free group.

Proof. We consider the action of $\mathrm{SL}_{2}(L(t))$ on the Bruhat-Tits tree $T_{v}$ for the discrete valuation on $L(t)$ at infinity, $v(f / g)=\operatorname{degree}(g)$ - degree $(f)$, for $f$ and $g$ in $L[t]$. The stabilizers of the vertices of $T_{v}$ are conjugates of $\mathrm{SL}_{2}(B)$, where $B$ is the valuation ring of $v$. It follows that the trace of an element of $\mathrm{SL}_{2}(L(t))$ that fixes a vertex is an element of $B$. Thus if an element of $\Gamma$ fixes a vertex, its trace is in $A \cap B$. An element of $A \cap B$ has the form $t^{-n} f(t)$ where $f(t)$ is a polynomial from $L[t]$ and $\operatorname{deg}(f) \leq n$ and thus $A \cap B=L\left[t^{-1}\right]$. Thus the trace of an element $\gamma \in \Gamma$ that stabilizes a vertex of the tree is of the form $r+\sigma(r)$, where $r \in A$ and, therefore, $r+\sigma(r) \in L\left[t^{-1}\right]$ and $r(1)=1$. Since this trace is invariant under $\sigma$, it must be constant, and hence by evaluation at $t=1$, it follows immediately that $\gamma$ is unipotent. We have thus shown that any subgroup of $\Gamma$ without unipotents acts freely on this tree so by Serre's theorem $[S$, p. 27] it is a free group.

Theorem 2. If $\mathscr{H}$ is a nonsplit quaternion algebra then $\Gamma$ is a free group.

Proof. As in the proof of Theorem 1, an element stabilizing a vertex of the tree yields elements $r, s$ such that $r \sigma(r)-\beta s \sigma(s)=1, r(1)=1, s(1)=0$, $\sigma(r)=2-r$. Hence $-\beta s \sigma(s)=(r-1)^{2}$ so that for $p=r-1, \beta s \sigma(s)=p \sigma(p)$. If $p$ is nonzero, we may multiply $s, p$ by suitable powers of $t$ so that they are polynomials in $t$, and then cancelling common powers of $t-1$ from each side of this equation obtain $p(1) / s(1) \in L$ has norm $\beta$. This contradicts the fact that the algebra is nonsplit [L, p. 58]. Thus $r=1$, so that any element of a stabilizer subgroup is trivial. Thus the group $\Gamma$ acts freely on the tree.

Let $F$ be a field of level $s(F) \geq 4$ [L, p. 302], that is, -1 is not a square or a sum of two squares of elements from $F$. In this case, the algebra with $\alpha=-1, \beta=-1$ is nonsplit. Let $L=F(i)$ be the extension of degree 2 over $F$, where $i^{2}=-1$.

Corollary. Let $L=F(i), s(F) \geq 4$. Then the group $\Gamma=\mathrm{SU}_{2}\left(L\left[t, t^{-1}\right], t-1\right)$ is a free group.

Proof. With involution $\sigma$ so that $i \rightarrow-i, t \rightarrow t^{-1}$, the group of norm one elements $\Gamma$ in this case is $\mathrm{SU}_{2}\left(L\left[t, t^{-1}\right], t-1\right)$, the kernel of the homomorphism

$$
\mathrm{SU}_{2}\left(L\left[t, t^{-1}\right]\right) \rightarrow \mathrm{SU}_{2}(L)
$$

where of course $\mathrm{SU}_{2}$ means the group of 2 by 2 matrices $X$, so that $\sigma(X)^{t}=$ $X^{-1}$. The result now follows immediately from Theorem 2 and the remarks above.

It would be interesting to know if Pollen's result that the matrices with linear entries form a set of free generators of this unitary group is also valid in this more general context. 


\section{REFERENCES}

[L] T. Y. Lam, Algebraic theory of quadratic forms, Benjamin, Reading, MA, 1973.

[P] D. Pollen, $\mathrm{SU}_{1}(2, F[z, 1 / z])$, J. Amer. Math. Soc. 3 (1990), 611-624.

[S] J.-P. Serre, Trees, Springer-Verlag, New York, 1980.

Department of Mathematics and Computer Science, San Jose State University, San Jose, CALIFORNIA 95192-0001 